\title{
ON SINGULAR $H$-CLOSED EXTENSIONS
}

\author{
KAVITA SRIVASTAVA
}

(Communicated by Richard Tall)

\begin{abstract}
Introducing the notion of singular $H$-closed extensions of a locally $H$-closed space, we obtain a necessary and sufficient condition for a semiregular $H$-closed extension to be singular.
\end{abstract}

\section{INTRODUCTION}

By a space we mean a Hausdorff space, and a map will mean a continuous map. Letters $X$ and $Y$ are used for spaces.

The concept of singular set of a mapping introduced by Cain [2] to study the relationship of compact mappings with other mappings is found useful in the study of compactifications of a locally compact space $[5,7,8,10]$. This concept which arose earlier in [16] led to the notion of singular compactifications. These have been further studied in [3-5, 9, 17]. In fact, the simple observation by Cain, Chandler, and Faulkner [5] that for a map $f$ from a locally compact space $X$ to a locally compact space $Y$, the remainder induced by $f$ (see [6]) and the singular set of $f$ are the same has led to a fruitful combination of the independent studies of both these concepts.

As is remarked in the treatise by Porter and Woods [15], that in many ways compact spaces are to Tychonoff spaces as $H$-closed spaces are to Hausdorff spaces, it is desirable to undertake the above-mentioned study in the context of $H$-closed extensions. In this spirit we introduce the concept of $H$-singular sets in $\S 2$ and use this to construct $H$-closed extensions of locally $H$-closed spaces in $\S 3$. Also, the notion of singular $H$-closed extensions is introduced in this section. We obtain a necessary and sufficient condition for a semiregular $\mathrm{H}$ closed extension of a locally $H$-closed space to be a singular $H$-closed extension in $\S 4$.

A space is said to be $H$-closed if it is closed in every Hausdorff space in which it can be embedded as a subspace, and a locally $H$-closed (LHC) space is a space in which each point has an $H$-closed neighborhood. An $H$-closed extension of a space $X$ is an $H$-closed space $Y$ in which $X$ is densely embedded; $Y-X$ is called the remainder of $X$ in $Y$. An open cover $\mathscr{U}$ of a space $X$ is said to

Received by the editors July 6, 1991.

1991 Mathematics Subject Classification. Primary 54D35, 54D40.

Key words and phrases. $H$-closed spaces, $H$-closed extensions, locally $H$-closed spaces, singular $H$-closed extensions, $p$-map, $p$-cover, remainder, retract. 
be a $p$-cover if there is a finite subfamily of $\mathscr{U}$ whose union is dense in $X$. A map $f$ from a space $X$ to a space $Y$ is said to be $p$-map if for each $p$-cover $\mathscr{U}$ of $Y, f^{-1}(\mathscr{U})=\left\{f^{-1}(U) \mid U \in \mathscr{U}\right\}$ is a $p$-cover of $X$. A space is called semiregular if the family of regular open sets of the space forms a base for its topology. An extensive account of $H$-closed spaces and $H$-closed extensions can be found in [13-15]. Also, we refer to [1,11] for other related studies.

For $A \subset X$, the closure of $A$ in $X$ will be denoted by $\mathrm{Cl}_{X} A$ or simply by $\mathrm{Cl} A$ if it is clear from the context. The family of $H$-closed sets of $X$ is denoted by $\mathscr{H}_{X}$.

The real number system is denoted by $R$ and the closed interval $[0,1]$ by $I$. The symbols $Q$ and $N$ denote the sets of rational numbers and natural numbers respectively. Unless stated otherwise, $R$ carries the usual topology and $I, Q$, and $N$ are treated as its subspaces.

Let $\tau$ be the topology on $X$ and $A \subset X$ be such that $A \notin \tau$. Then $X$ equipped with the topology generated by $\tau \cup \tau_{1}$, where $\tau_{1}=\{\varnothing, A, X\}$ is called the simple extension of $X$ by $A$ [18].

\section{2. $H$-SINGULAR SETS}

2.1. Definition. Let $f$ be a map from $X$ to $Y$. Then the $H$-singular set $S_{h}(f)$ of $f$ is the set $\left\{y \in Y \mid\right.$ for every open set $U$ of $Y$ containing $y, f^{-1}(U)$ is not contained in an $H$-closed set of $X\}$.

Note that

$$
\begin{aligned}
S_{h}(f)= & \{y \in Y \mid \text { for every open set } U \text { of } Y \text { containing } y, \\
& \left.\mathrm{Cl} f^{-1}(U) \text { is not contained in an } H \text {-closed set of } X\right\} \\
= & \{y \in Y \mid \text { for every open set } U \text { of } Y \text { containing } y, \\
& \left.\quad f^{-1}(U) \text { is not } H \text {-closed }\right\} .
\end{aligned}
$$

2.2. Proposition. For a map $f$ from $X$ to $Y$,

$$
S_{h}(f)=\bigcap_{H \in \mathscr{H}_{X}} \mathrm{Cl} f(X-H) .
$$

Proof. The proof is straightforward.

2.3. Proposition. Let $f$ be a map from a space $X$ to the real line $R$. If $f$ has the extension $H f$ to the $H$-closed extension $H X$ of $X$ and if $H X-X$ is $H$-closed, then $H f(H X-X)=S_{h}(f)$.

Proof. Noting that, for $H \in \mathscr{H}_{X}, H X-X \subset \mathrm{Cl}_{H X}(X-H)$ and hence $H f(H X-X) \subset H f\left(\mathrm{Cl}_{H X}(X-H)\right) \subset \mathrm{Cl} H f(X-H)=\mathrm{Cl} f(X-H)$, we obtain $H f(H X-X) \subset S_{h}(f)$ in view of Proposition 2.2. Since $H f(H X-X)$ is closed, to complete the proof it suffices to show that $H f(H X-X)$ is dense in $S_{h}(f)$. If otherwise, then there exists a $p \in S_{h}(f)$ and an open set $U$ in $R$ containing $p$ such that $\mathrm{Cl} U \cap H f(H X-X)=\varnothing$, so that

$$
(H f)^{-1}(\mathrm{Cl} U) \subset(H f)^{-1}(R-H f(H X-X)) \subset X,
$$

which gives that $\mathrm{Cl}_{X} f^{-1}(U)=\mathrm{Cl}_{H X}(H f)^{-1}(U)$ is $H$-closed in $X$. This contradicts that $p \in S_{h}(f)$. 


\section{Singular $H$-CLOSED EXTENSIONS}

Let $f$ be a $p$-map from an LHC space $X$ to an $H$-closed space $Y$ such that $f(X)$ is dense in $Y$. Topologize the disjoint union $X \cup Y$ of $X$ and $Y$ as follows: Each open set of $X$ is open in $X \cup Y$ and, for $y$ in $Y$, the family $\left\{U \cup\left(f^{-1}(U)-H\right) \mid U\right.$ is open in $Y, y \in U$ and $\left.H \in \mathscr{H}_{X}\right\}$ forms a neighborhood base. With this topology $X \cup Y$ is an $H$-closed space. That it is a Hausdorff space can be verified by using local $H$-closedness of $X$. To prove that $X \cup Y$ is $H$-closed, let $\vartheta=\left\{V_{a} \mid a \in \mathscr{A}, \mathscr{A}\right.$ is an index set $\}$ be an open cover of $X \cup Y$ by basic open sets. Then there exists a subset $\mathscr{B}$ of $\mathscr{A}$ such that, for $b$ in $\mathscr{B}, V_{b}=U_{b} \cup\left(f^{-1}\left(U_{b}\right)-H_{b}\right)$, where $U_{b}$ is open in $Y, H_{b} \in \mathscr{H}_{X}$, and $\left\{U_{b} \mid b \in \mathscr{B}\right\}$ is a $p$-cover of $Y$. Thus $\left\{f^{-1}\left(U_{b}\right) \mid b \in\right.$ $\mathscr{B}\}$ is a $p$-cover of $X$. Therefore, there exists a finite set $\mathscr{C}$ of $\mathscr{B}$ such that $\bigcup_{c \in \mathscr{C}} \mathrm{Cl}_{X} f^{-1}\left(U_{c}\right)=X$. Since $\bigcup_{c \in \mathscr{C}}\left(\mathrm{Cl}_{X} f^{-1}\left(U_{c}\right)-H_{c}\right)$ is contained in $\bigcup_{c \in \mathscr{C}} \mathrm{Cl}_{X}\left(f^{-1}\left(U_{c}\right)-H_{c}\right), \bigcup_{c \in \mathscr{C}} \mathrm{Cl}_{X \cup Y}\left(f^{-1}\left(U_{c}\right)-H_{c}\right)$ contains $X-\bigcup_{c \in \mathscr{C}} H_{c}$. As $\bigcup_{c \in \mathscr{C}} H_{c}$ is $H$-closed in $X \cup Y$, there exists a finite set $\mathscr{D}$ contained in $\mathscr{A}$ such that $\bigcup_{c \in \mathscr{C}} H_{c} \subset \bigcup_{d \in \mathscr{D}} \mathrm{Cl}_{X \cup Y} V_{d}$. Further, the $p$-cover $\left\{U_{b} \mid b \in \mathscr{B}\right\}$ of $Y$ provides a finite set $\mathscr{E}$ in $\mathscr{B}$ such that $Y \subset \bigcup_{e \in \mathscr{E}} \mathrm{Cl}_{X \cup Y} V_{e}$. Now, it follows that the union of members of $\vartheta$ corresponding to the finite set $\mathscr{C} \cup \mathscr{D} \cup \mathscr{E}$ of $\mathscr{A}$ is dense in $X \cup Y$.

Noting that $\mathrm{Cl}_{X \cup Y} X=X \cup S_{h}(f)$, we find that $X \cup S_{h}(f)$ is an $H$-closed extension of $X$, and thus we have established the following theorem.

3.1. Theorem. If $f$ is a p-map from a locally $H$-closed space $X$ to an $H$-closed space $Y$ such that $f(X)$ is dense in $Y$, then $X$ has an $H$-closed extension with $S_{h}(f)$ as the remainder.

3.2. Remark. Let $f$ be a map from a locally compact space $X$ to a compact space $Y$ such that $f(X)$ is dense in $Y$. Then the singular set $S(f)$ of $f$ is the set $\left\{y \in Y \mid\right.$ for every open set $U$ of $Y$ containing $y, \mathrm{Cl}^{-1}(U)$ is not compact\}. In this case $S_{h}(f)=S(f)$ and $X \cup S_{h}(f)$ coincides with the singular compactification $X \cup S(f)$ of $X$. It may be noted that, since $Y$ is compact, the map $f$ is a $p$-map.

The following example shows that if $f$ is not a $p$-map, then the method described may not give an $H$-closed extension.

3.3. Example. Denote by $I_{d}$ the closed interval $[0,1]$ of $R$ with the discrete topology, and let $I_{Q}$ be the simple extension of $I$ by $I \cap Q$. Then $I_{Q}$ is an $H$-closed space. Consider the identity map $f$ from $I_{d}$ to $I_{Q}$. It is clear that $S_{h}(f)=I_{Q}$ and $f$ is not a $p$-map. The space $I_{d} \cup I_{Q}$ fails to be $H$ closed because the open cover $\left\{(1 /(n+1), 1 / n) \cup f^{-1}(1 /(n+1), 1 / n) \mid n \in\right.$ $N\} \cup\left\{(I \cap Q) \cup f^{-1}(I \cap Q)\right\}$ is not a $p$-cover.

3.4. Proposition. Let $f$ be a p-map from a locally $H$-closed space $X$ to an $H$-closed space $Y$ such that $S_{h}(f)=Y$. Then $X$ has an $H$-closed extension with $Y$ as the remainder.

Proof. The proof follows immediately from Theorem 3.1.

3.5. Definition. The $H$-closed extension $X \cup S_{h}(f)$ of a locally $H$-closed space $X$, where $f$ is a $p$-map from $X$ to an $H$-closed space $Y$ such that $S_{h}(f)=Y$, is called a singular $H$-closed extension of $X$.

We give below some examples of singular $H$ closed extensions. 
3.6. Example. The constant map from a non- $H$-closed LHC space $X$ to the one-point space provides a singular $H$-closed extension of $X$ with remainder as the one-point space. Obviously for an LHC space, a singular $H$-closed extension with one-point space as the remainder is unique.

3.7. Example. Consider a map $f$ from $N$ to $I_{Q}$ which is injective and satisfies $f(N)=I \cap Q$. The map $f$ is a $p$-map with $S_{h}(f)=I_{Q}$. This provides a singular $H$-closed extension of $N$ with $I_{Q}$ as the remainder.

3.8. Example. Let $D$ be the discrete space of cardinality $c$ and $I_{Q^{\prime}}$ be the simple extension of $I$ by $I \cap(R-Q)$. Define $f$ from $D$ to $I_{Q^{\prime}}$ which is injective and satisfies $f(D)=I \cap(R-Q)$. The map $f$ is a $p$-map with $S_{h}(f)=I_{Q^{\prime}}$. This gives a singular $H$-closed extension of $D$ with $I_{Q^{\prime}}$ as the remainder.

\section{Singular $H$-ClOSED EXTENSIONS AND RETRACTS}

Guglielmi [12] obtained that a compactification $\alpha X$ of a locally compact space $X$ is singular iff $\alpha X-X$ is a retract of $\alpha X$. An analogue of this result may be expected in the case of a singular $H$-closed extension of an LHC space. In this section we obtain that this is true under the condition when the extension is semiregular. We provide an example to show the need for this condition.

4.1. Theorem. A semiregular $H$-closed extension $H X$ of a locally $H$-closed space $X$ is singular iff $H X-X$ is a retract of $H X$.

Proof. If $H X$ is a singular $H$-closed extension with respect to a map $f: X \rightarrow$ $H X-X$, then the map $r: H X \rightarrow H X-X$ defined by $r(x)=x$, if $x \in H X-X$ and $r(x)=f(x)$, if $x \in X$, is a retraction. Conversely, suppose that $r: H X \rightarrow$ $H X-X$ is a retraction and $f$ is the restriction of $r$ to $X$. Because the inclusion of $X$ into $H X$ and $r$ are $p$-maps, $f$ is also a $p$-map. To show that $S_{h}(f)=H X-X$, let, for $p \in H X-X, U$ be an open set of $H X-X$ containing $p$. Since $X$ is dense in $H X$ and $r^{-1}(U)$ is an open neighbourhood of $p, p$ is necessarily in $\mathrm{Cl}_{H X}\left(X \cap r^{-1}(U)\right)=\mathrm{Cl}_{H X}\left(f^{-1}(U)\right)$. But $p$ is not in $X$ and therefore not in $\mathrm{Cl}_{X}\left(f^{-1}(U)\right)$, implying that $\mathrm{Cl}_{X}\left(f^{-1}(U)\right) \neq \mathrm{Cl}_{H X}\left(f^{-1}(U)\right)$, and thus $\mathrm{Cl}_{X} f^{-1}(U)$ is not $H$-closed. Therefore $p \in S_{h}(f)$. Since for an open set $U$ of $H X-X$ and $H \in \mathscr{H}_{X}, h^{-1}\left(U \cup\left(f^{-1}(U)-H\right)\right)=r^{-1}(U)-H$, where $h: H X \rightarrow X \cup S_{h}(f)$ is the identity map, $h$ is continuous. Further, since $H X$ is semiregular, $h$ is closed as well. Hence $H X$ is a singular $H$-closed extension of $X$.

The following example shows that an $H$-closed extension $H X$ of an LHC space $X$ which is not semiregular is not necessarily a singular $H$-closed extension even if $H X-X$ is a retract of $H X$.

4.2. Example. Consider the subspace

$$
X=\left\{\left(\frac{1}{n}, \frac{1}{m}\right) \mid n, m \in N\right\} \cup\left\{\left(\frac{1}{n}, 0\right) \mid n \in N\right\}
$$

of the Euclidean plane $R^{2}$. Let $Y=X \cup\{p\}$, where $p$ is not in $X$. Equip $Y$ with a topology such that $U \subset Y$ is open provided that $U \cap X$ is open in $X$ and if $p \in U$, then there exists a $k \in N$ such that $\left\{\left(\frac{1}{n}, \frac{1}{m}\right) \mid n \geq k\right.$ 
and $m \in N\} \subset U$. Then $Y$ is an $H$-closed extension of $X$ which is not semiregular $[15,7.3(\mathrm{~d})]$. Obviously, $Y-X=\{p\}$ is a retract of $Y$. In view of Remark 3.2, the singular $H$-closed extension of $X$ with remainder as the one-point space is one-point compactification of $X$. Since $Y$ and the onepoint compactification of $X$ are not homeomorphic [15, 7.3(d)], $Y$ is not a singular $H$-closed extension of $X$.

4.3. Remark. From the proof of Theorem 4.1, it is clear that if $H X$ is a singular $H$-closed extension of an LHC space $X$, then $H X-X$ is a retract of $H X$. However, a singular $H$-closed extension need not be semiregular: The fact that a space which is not semiregular cannot have a semiregular $H$ closed extension provides examples. Specifically, the one-point singular $H$ closed extension of the simple extension $R_{Q}$ of $R$ by $Q$ is not semiregular.

\section{ACKNOWLEDGMENTS}

The author expresses her gratefulness to Dr. K. K. Azad for his continued encouragement and valuable suggestions during the preparation of this manuscript.

The author thanks the referee for giving some significant suggestions, particularly for providing a simpler argument for a statement in the proof of Theorem 4.1.

\section{REFERENCES}

1. B. Banaschewski, Extensions of topological spaces, Canad. Math. Bull. 7 (1964), 1-22.

2. George L. Cain, Jr., Compact and related mappings, Duke Math. J. 33 (1966), 639-645.

3. - Compactness of certain closed mappings, Notices Amer. Math. Soc. 14 (1967), 374.

4. __ Mappings with prescribed singular sets, Nieuw Arch. Wisk. (4) 17 (1969), 200-203.

5. George L. Cain, Jr., Richard E. Chandler, and Gary D. Faulkner, Singular sets and remainders, Trans. Amer. Math. Soc. 268 (1981), 161-171.

6. Richard E. Chandler and Fu-Chien Tzung, Remainders in Hausdorff compactifications, Proc. Amer. Math. Soc. 70 (1978), 196-202.

7. Richard E. Chandler and Gary D. Faulkner, Singular compactifications: the order structure, Proc. Amer. Math. Soc. 100 (1987), 377-382.

8. Richard E. Chandler, Gary D. Faulkner, Josephine P. Guglielmi, and Margaret C. Memory, Generalizing the Alexandroff-Urysohn double circumference construction, Proc. Amer. Math. Soc. 83 (1981), 606-608.

9. R. F. Dickman, Unified spaces and singular sets for mappings, Fund. Math. 62 (1968), 103-123.

10. Gary D. Faulkner, Compactifications whose remainders are retracts, Proc. Amer. Math. Soc. 103 (1988), 984-988.

11. M. Girou, Properties of locally H-closed spaces, Proc. Amer. Math. Soc. 113 (1991), 287-295.

12. Josephine P. Guglielmi, Compactifications with singular remainders, $\mathrm{Ph} . \mathrm{D}$. Thesis, North Carolina State Univ., 1986.

13. J. R. Porter and C. Votaw, H-closed extensions. I, Topology Appl. 3 (1973), 211-224.

14. __ H-closed extensions. II, Trans. Amer. Math. Soc. 202 (1975), 193-209.

15. J. R. Porter and R. G. Woods, Extensions and absolutes of Hausdorff spaces, Springer-Verlag, New York, Berlin, Heidelberg, London, Paris, and Tokyo, 1988. 
16. G. T. Whyburn, On compactness of mappings, Proc. Nat. Acad. Sci. U.S.A. 52 (1964), 1426-1431.

17. _ـ Compactifications of mappings, Math. Ann. 166 (1966), 168-174.

18. A. Wilansky, Topology for analysis, Ginn, Waltham, MA, Toronto and London, 1970.

Department of Mathematics and Statistics, University of Allahabad, 211002 AllaHABAD, INDIA

Mailing address: Vijaya Niwas, 198, Mumfordganj, Allahabad-211 002, India 